\title{
On High-power Operator Inequalities and Spectral Radii of Operators
}

\author{
By \\ Chia-Shiang LiN* and Sever Silvestru DRAGOMIR**
}

\begin{abstract}
For some different types of operators on a Hilbert space, we present new highpower operator inequalities, and their corresponding operator inequalities involving spectral radii of operators. In particular, we show that Halmos' two operator inequalities, Reid's inequality and many others hold easily. In applications we obtain a new generalized classical Löwner inequality; and a slightly generalized Löwner-Heinz inequality is given.
\end{abstract}

\section{$\S 1 . \quad$ Introduction}

As is well-known the Cauchy-Schwarz inequality is a powerful inequality which states that the relation

$$
|(x, y)| \leq\|x\|\|y\|
$$

holds for every $x$ and $y$ in a pre-Hilbert space. Every inequality in this space is either derived from the Cauchy-Schwarz inequality, or equivalent to it. For recent developments on inequalities related to (1.1) see [1] and the references therein.

Communicated by H. Okamoto. Received November 12, 2004. Revised February 4, 2005. 2000 Mathematics Subject Classification(s): 47A63

Key words: Cauchy-Schwarz inequality, high-power operator inequality, spectral radius of operator, positive operator, Reid's inequality, Löwner inequality, and Löwner-Heinz inequality.

*Department of Mathematics, Bishop's University, Lennoxville, Quebec, J1M 1Z7, Canada.

e-mail: plin@ubishops.ca

${ }^{* *}$ School of Computer and Mathematics, Victoria University of Technology, PO Box 14428, MCMC 8001, Victoria, Australia.

e-mail: sever@csm.vu.edu.au

(C) 2006 Research Institute for Mathematical Sciences, Kyoto University. All rights reserved. 
In this paper we use capital letters to denote bounded linear operators on a complex Hilbert space $H$ into itself, and $I$ denotes the identity operator. A positive operator $T$ is written as $T \geq O$, the zero operator. We shall consider four types of high-power operator inequalities, and their corresponding operator inequalities involving spectral radii of operators. Four types are: a positive operator, two arbitrary operators, mixed operators, and two selfadjoint operators. Indeed, our results are motivated by Halmos' two operator inequalities in [3, p. 51 and 244]. He proved that if $T \geq O, S$ is arbitrary and $T S$ is selfadjoint operators, then the following high-power operator inequality holds.

$$
|(T S x, x)|^{2^{n}} \leq\left(T S^{2^{n}} x, x\right)(T x, x)^{2^{n}-1}
$$

for every $x \in H$ and $n \geq 0$. From this he concluded that the inequality involving spectral radius

$$
|(T S x, x)| \leq r(S)(T x, x)
$$

holds, where $r(S)$ means the spectral radius of $S$. It is a stronger version of a result due to Reid [9]; Reid had $\|S\|$ instead of $r(S)$ (that $r(S) \leq\|S\|$ is known [3, p. 45]). Actually, we obtain some generalizations of inequalities (1.2) and (1.3). In particular, it is shown that inequalities (1.2), (1.3), Reid's inequality, and many others hold easily. In applications we obtain a new generalized classical Löwner inequality, and partially generalized Löwner-Heinz inequality. The latter, as well-known, is essential in general operator inequalities on $H$.

\section{$\S 2 . \quad$ Results}

First of all, recall that the inequality $|(T x, y)|^{2} \leq(T x, x)(T y, y)$ holds for $T \geq O$ and for all $x, y \in H$ (consider the unique positive square root of $T)$. In fact, it is known to be equivalent to the Cauchy-Schwarz inequality, and this is crucial in the proof of our results. Next, we need a well known relation: $r(S)=\lim _{n}\left\|S^{n}\right\|^{1 / n}$ for any operator $S$ [3, Problem 74].

Lemma 1. Let $T \geq O$, and $S$ and $C$ be arbitrary operators. Also let $T S, T C, A$ and $B$ be all selfadjoint operators. If $n$ is a positive integer, then for all $x, y \in H$ the following hold true.

$$
\begin{aligned}
|(T x, y)|^{2^{n} \leq} \leq & \left(T^{1+2^{n-1}} x, x\right)(T x, x)^{2^{n-1}-1}\|y\|^{2^{n}}, \quad n \geq 1 . \\
|(T S x, C y)|^{2^{n} \leq} & \left(T S^{2^{n}} x, x\right)(T x, x)^{2^{n-1}-1} \\
& \times\left(T C^{2^{n}} y, y\right)(T y, y)^{2^{n-1}-1}, \quad n \geq 1 . \\
|(A x, B y)|^{2^{n} \leq} & \left(A^{2^{n-1}+2} x, x\right)\left(B^{2^{n-1}+2} y, y\right)\|A x\|^{2^{n-1}-2} \\
& \times\|B y\|^{2^{n-1}-2}\|x\|^{2^{n-1}}\|y\|^{2^{n-1}}, n \geq 2 .
\end{aligned}
$$


Proof. (2.1). We shall prove it inductively. For $n=1,|(T x, y)|^{2} \leq$ $\left(T^{2} x, x\right)\|y\|^{2}$. As $\left(T^{2} x, x\right)^{2} \leq(T T x, T x)(T x, x)=\left(T^{3} x, x\right)(T x, x)$, we have

$$
|(T x, y)|^{4} \leq\left(T^{2} x, x\right)^{2}\|y\|^{4} \leq\left(T^{3} x, x\right)(T x, x)\|y\|^{4}
$$

for $n=2$. Since

$$
\left(T^{1+2^{n-1}} x, x\right)^{2} \leq\left(T T^{2^{n-1}} x, T^{2^{n-1}} x\right)(T x, x)=\left(T^{1+2^{n}} x, x\right)(T x, x),
$$

we obtain

$$
\begin{aligned}
& |(T x, y)|^{2^{n+1}} \leq\left[|(T x, y)|^{2^{n}}\right]^{2} \\
& \leq\left[\left(T^{1+2^{n-1}} x, x\right)(T x, x)^{2^{n-1}-1}\|y\|^{2^{n}}\right]^{2} \leq\left(T^{1+2^{n}} x, x\right)(T x, x)^{2^{n}-1}\|y\|^{2^{n+1}}
\end{aligned}
$$

and the induction process is completed.

(2.2). As $T$ is positive and both $T S$ and $T C$ are selfadjoint, we see that $S^{*} T S=(T S)^{*} S=T S^{2}$. And by induction we get $\left(S^{*}\right)^{i} T S^{i}=T S^{2 i}, i=$ $1,2, \ldots$ Similarly, $\left(C^{*}\right)^{i} T C^{i}=T C^{2 i}, i=1,2, \ldots$ It follows, for $n=1$, that

$$
|(T S x, C y)|^{2} \leq(T S x, S x)(T C y, C y)=\left(T S^{2} x, x\right)\left(T C^{2} y, y\right) .
$$

Since $\left(T S^{2^{n}} x, x\right)^{2} \leq\left(\left(S^{*}\right)^{2 n} T S^{2^{n}} x, x\right)(T x, x)=\left(T S^{2^{n+1}} x, x\right)(T x, x)$, we have

$$
\begin{aligned}
& |(T S x, C y)|^{2^{n+1}} \leq\left(T{S^{2^{n}}} x, x\right)^{2}(T x, x)^{2^{n}-2}\left(T C^{2^{n}} y, y\right)^{2}(T y, y)^{2^{n}-2} \\
& \leq\left(T S^{2^{n+1}} x, x\right)(T x, x)^{2^{n}-1}\left(T C^{2^{n+1}} y, y\right)(T y, y)^{2^{n}-1} .
\end{aligned}
$$

This proves, by induction, the inequality (2.2).

(2.3). Since $|(A x, B y)|^{2} \leq\left(A^{2} x, x\right)\left(B^{2} y, y\right)$,

$$
\begin{aligned}
& |(A x, B y)|^{4} \leq\left(A^{2} x, x\right)^{2}\left(B^{2} y, y\right)^{2} \\
& \leq\left(A^{2} x, A^{2} x\right)\left(B^{2} y, B^{2} y\right)\|x\|^{2}\|y\|^{2}=\left(A^{4} x, x\right)\left(B^{4} y, y\right)\|x\|^{2}\|y\|^{2}
\end{aligned}
$$

for $n=2$. Note that $A^{2} \geq O$ as $A$ is selfadjoint, and

$$
\left(A^{2^{n-1}+2} x, x\right)^{2}=\left(A^{2} A^{2^{n-1}} x, x\right)^{2} \leq\left(A^{2^{n}+2} x, x\right)\|A x\|^{2},
$$

and similarly for $B^{2} \geq O$. Therefore,

$$
|(A x, B y)|^{2^{n+1}} \leq\left(A^{2^{n}+2} x, x\right)\left(B^{2^{n}+2} y, y\right)\|A x\|^{2^{n}-2}\|B y\|^{2^{n}-2}\|x\|^{2^{n}}\|y\|^{2^{n}},
$$

and (2.3) holds by induction, and the proof of Lemma 1 is now completed. 
Theorem 1. Let $T \geq O$, and $S$ and $C$ be arbitrary operators. Also let $T S, T C, A$ and $B$ be all selfadjoint operators. If $n$ is a positive integer, then for all $x, y \in H$ the following hold true.

$$
\begin{aligned}
|(T x, y)|^{2} & \leq r(T)(T x, x)\|y\|^{2} . \\
|(T S x, C y)| & \leq r(S) r(C)(T x, x)^{1 / 2}(T y, y)^{1 / 2} . \\
|(T S x, C x)| & \leq r(S) r(C)(T x, x) . \\
|(A x, B y)|^{2} & \leq r(A) r(B)\|A x\|\|B y\|\|x\|\|y\| .
\end{aligned}
$$

Proof. (2.4). The inequality (2.1) in Lemma 1 gives

$$
|(T x, y)|^{2^{n}} \leq\|T\|\left\|T^{2^{n-1}}\right\|\|x\|^{2}(T x, x)^{2^{n-1}-1}\|y\|^{2^{n}} .
$$

Taking the $2^{n-1}$-th root of both sides of the inequality above yields

$$
|(T x, y)|^{2} \leq\|T\|^{\frac{1}{2^{n-1}}}\left\|T^{2^{n-1}}\right\| \frac{1}{2^{n-1}}\|x\|^{\frac{2}{2^{n-1}}}(T x, x)^{1-\frac{1}{2^{n-1}}}\|y\|^{2},
$$

and passing to the limit as $n \rightarrow \infty$ we have the desired conclusion.

(2.5). Note that (2.2) in Lemma 1 yields

$$
|(T S x, C y)|^{2^{n}} \leq\|T\|^{2}\left\|S^{2^{n}}\right\|\left\|C^{2^{n}}\right\|\|x\|^{2}\|y\|^{2}(T x, x)^{2^{n-1}-1}(T y, y)^{2^{n-1}-1},
$$

which implies, by taking the $2^{n}$-th root,

$$
\begin{aligned}
& |(T S x, C y)| \\
& \leq\|T\| \frac{1}{2^{n-1}}\left\|S^{2^{n}}\right\| \frac{1}{2^{n}}\left\|C^{2^{n}}\right\| \frac{1}{2^{n}}\|x\|^{\frac{1}{2^{n-1}}}\|y\| \frac{1}{2^{n-1}}(T x, x)^{\frac{1}{2}-\frac{1}{2^{n}}}(T y, y)^{\frac{1}{2}-\frac{1}{2^{n}}} .
\end{aligned}
$$

Thus, we have the inequality (2.5) after passing to the limit as $n \rightarrow \infty$.

(2.6) follows by setting $y=x$ in (2.5) above.

(2.7). The inequality (2.3) in Lemma 1 gives

$$
\begin{aligned}
& |(A x, B y)|^{2^{n}} \leq\|A\|^{2}\left\|A^{2^{n-1}}\right\|\|A x\|^{2^{n-1}-2}\|B\|^{2}\left\|B^{2^{n-1}}\right\| \\
& \times\|B y\|^{2^{n-1}-2}\|x\|^{2^{n-1}+2}\|y\|^{2^{n-1}+2} .
\end{aligned}
$$

The next step is taking the $2^{n-1}$-th root, and then passing to the limit as $n \rightarrow \infty$; the same as we did many times before. This proves Theorem 1 .

By a well-known result, if $E$ is a normal operator (selfadjoint operator, in particular) on a complex Hilbert space, then $r(E)=\|E\|[10$, Theorem 6.2-E]. Thus, the proofs of (2.4) and (2.7) in Theorem 1 are easy. However, each of our proof is a consequence of taking the limit of a high-power operator inequality, and does not rely on the result above. It should be pointed out that inequalities (2.2) and (2.5) are generalizations of Halmos' inequalities (1.2) and (1.3), respectively. 


\section{§3. Applications}

The classical Löwner-Heinz inequality was initiated in [7] and established in [8], which is a basic tool in theory of operator inequalities on $H$. More precisely, the inequality $P^{\alpha} \geq Q^{\alpha}$ holds if $P \geq Q \geq O$, where $\alpha \in[0,1]$. There are known examples showing that the inequality does not hold in general if $\alpha>1$. The proof of the inequality was neither elementary nor short. It should be mentioned here that Furuta gave an excellent and useful generalization of the Löwner-Heinz inequality in [2], and is called the Furuta inequality in the literature. There is a classical characterization of the Löwner-Heinz inequality, namely $P^{1 / 2} \geq Q^{1 / 2}$ holds if $P \geq Q \geq O$, which is known as the Löwner inequality. The inequality will be generalized later without relying on the Löwner inequality itself. First of all we have a partially generalized LöwnerHeinz inequality next.

Theorem 2. If $P \geq Q \geq O$, both $P^{\alpha} C$ and $C^{*} Q^{\alpha}$ are selfadjoint for some operator $C$ and $\alpha \in\left[0, \frac{1}{2}\right]$, then

$$
r(C) P^{\alpha} \geq C^{*} Q^{\alpha} .
$$

Proof. We show that the generalized Halmos' inequality (2.6) in Theorem 1 implies the required inequality. We may assume without loss of generality that $P$ is invertible, then $P^{-\alpha} Q^{2 \alpha} P^{-\alpha} \leq I$, Because the condition $P \geq Q \geq O$ implies that $P^{2 \alpha} \geq Q^{2 \alpha} \geq O$ for $\alpha \in\left[0, \frac{1}{2}\right]$ by the Löwner-Heinz inequality. Let $S=P^{-\alpha} Q^{\alpha}$. Then $S S^{*}=P^{-\alpha} Q^{2 \alpha} P^{-\alpha} \leq I$, i.e., $S$ is a contraction. Next, let $T=P^{\alpha} \geq O$, then $C^{*} T S=C^{*} Q^{\alpha}$. As both $P^{\alpha} C$ and $C^{*} Q^{\alpha}$ are selfadjoint by assumption (thus, $T \geq O$, and both $T S$ and $T C$ are selfadjoint), it follows from the inequality $|(T S x, C x)| \leq r(S) r(C)(T x, x)$ which is $(2.6)$ in Theorem 1 that

$$
\left(C^{*} Q^{\alpha} x, x\right) \leq r(S)\left(r(C) P^{\alpha} x, x\right) \leq\left(r(C) P^{\alpha} x, x\right)
$$

for every $x \in H$, and we have (3.1).

Remark that we use both the Löwner-Heinz inequality and the inequality (2.6) to prove Theorem 2. However, the next result is a generalized Löwner inequality without using the Löwner inequality itself.

Corollary 1. If $P \geq Q \geq O$, both $P^{1 / 2} C$ and $C^{*} Q^{1 / 2}$ are selfadjoint for some operator $C$, then

$$
r(C) P^{1 / 2} \geq C^{*} Q^{1 / 2}
$$


Proof. The inequality (3.2) is obtained by letting $\alpha=1 / 2$ in the proof of Theorem 2.

The next result shows different high-power operator inequalities.

Corollary 2. If $P \geq Q \geq O$, both $P^{\alpha} C$ and $C^{*} Q^{\alpha}$ are selfadjoint for some operator $C$ and $\alpha \in\left[0, \frac{1}{2}\right]$, then, for all $x, y \in H$, we have

$$
\begin{aligned}
\left|\left(C^{*} Q^{\alpha} x, y\right)\right|^{2^{n} \leq} & \left(P^{\alpha}\left(P^{-\alpha} Q^{\alpha}\right)^{2^{n}} x, x\right)\left(P^{\alpha} x, x\right)^{2^{n-1}-1}\left(P^{\alpha} C^{2^{n}} y, y\right) \\
& \times\left(P^{\alpha} y, y\right)^{2^{n-1}-1}, \quad n \geq 1 . \\
\left|\left(C^{*} Q^{\alpha} x, y\right)\right| \leq & r(C)\left(P^{\alpha} x, x\right)^{1 / 2}\left(P^{\alpha} y, y\right)^{1 / 2} . \\
\left(C^{*} Q^{\alpha} x, x\right)^{2^{n} \leq} & \left(P^{\alpha}\left(P^{-\alpha} Q^{\alpha}\right)^{2^{n}} x, x\right)\left(P^{\alpha} x, x\right)^{2^{n}-2} \\
& \times\left(P^{\alpha} C^{2^{n}} x, x\right), \quad n \geq 1 . \\
\left|\left(Q^{\alpha} x, y\right)\right|^{2^{n} \leq} & \left(P^{\alpha}\left(P^{-\alpha} Q^{\alpha}\right)^{2^{n}} x, x\right)\left(P^{\alpha} x, x\right)^{2^{n-1}-1} \\
& \times\left(P^{\alpha} y, y\right)^{2^{n-1}}, \quad n \geq 1 . \\
\left|\left(Q^{\alpha} x, x\right)\right|^{2^{n} \leq} & \left(P^{\alpha}\left(P^{-\alpha} Q^{\alpha}\right)^{2^{n}} x, x\right)\left(P^{\alpha} x, x\right)^{2^{n}-1}, \quad n \geq 1 .
\end{aligned}
$$

Proof. In the inequalities (2.2) and (2.5) in section 1 let $T=P^{\alpha} \geq O$ and $S=P^{-\alpha} Q^{\alpha}$. Then $S$ is a contraction, $T S$ and $T C$ are selfadjoint as in the proof of Theorem 2; and inequalities (3.3) and (3.4), respectively, follow. (3.5) follows by setting $y=x$ in (3.3) above. Finally, (3.6) and (3.7) are particular cases of (3.3) and (3.5), respectively, where $C=I$.

For the next result let $E=U|E|$ be the polar decomposition of the operator $E$ with $U$ the partial isometry, and $|E|$ the positive square root of the positive operator $E^{*} E$.

Corollary 3. Let $T \geq O$ and $T S$ be a selfadjoint operator. Then the following are equivalent.

(3.8) $(|T S| x, x) \mid \leq\|S\|(T x, x)$ for every $x \in H$;

(3.9) $|(T S x, x)| \leq\|S\|(T x, x)$ for every $x \in H$ (Reid's inequality);

$$
P^{1 / 2} \geq Q^{1 / 2} \text { if } P \geq Q \geq O \text { (Löwner inequality). }
$$

Proof. We use a familiar relation that $-|A| \leq A \leq|A|$ holds if $A$ is selfadjoint. In other words, $|(A x, x)| \leq(|A| x, x)$ for every $x \in H$. Hence, (3.8) implies (3.9).

$(3.9) \Rightarrow(3.10)$. In the proof of Theorem 2 let $C=I, \alpha=\frac{1}{2}$ and use the inequality $|(T S x, C x)| \leq\|S\|\|C\|(T x, x)$ instead of $(2.6)$. 
$(3.10) \Rightarrow(3.8)$. Since $S /\|S\|$ is a contraction, i.e., $S S^{*} \leq\|S\|^{2} I$, we have

$$
O \leq(T S)^{2}=T S(T S)^{*}=T S S^{*} T \leq\|S\|^{2} T^{2} .
$$

It follows that $|T S| \leq\|S\| T$ and we have (3.8).

Finally, we remark that the equivalence of the Reid's inequality and the Löwner-Heinz inequality has been pointed out in [11].

\section{References}

[1] Dragomir, S. S., Advances in Inequalities of the Schwarz, Gruss and Bessel Type in Inner Product Spaces, Preprint available on line at: http://front.math.ucdavis.edu/ math.FA/0309354.

[2] Furuta, T., $A \geq B \geq O$ assures $\left(B^{r} A^{p} B^{r}\right)^{1 / q} \geq B^{(p+2 r) / q}$ for $r \geq 0, p \geq 0, q \geq 1$ with $(1+2 r) q \geq p+2 r$, Proc. Amer. Math. Soc., 101 (1987), 85-88.

[3] Halmos, P. R., Hilbert Space Problem Book, Van Nostrand, Princeton, NJ, 1967.

[4] Lin, C.-S., Operator versions of inequalities and equalities on a Hilbert space, Lin. Alge. Its Appli., 268 (1998), 365-374.

[5] On Halmos' sharpening of Reid's inequality, C. R. Math. Acad. Sci. Soc. R. Can., 20 (1998), 62-64.

[6] Polar decomposition approach to Reid's inequality, J. Inequal. Appl., 7 (2002), 779-785.

[7] Löwner, K., Über monotone Matrixfunktionen, Math. Z., 38 (1934), 177-216.

[8] Heinz, E., Beiträge zur Störungstheorie der Spektralzerlegung, ibid., 123 (1951), 415438

[9] Reid, W. T., Symmetrizable completely continuous linear transformations in Hilbert space, Duke Math. J., 18 (1951), 41-56.

[10] Taylor, A. E., Introduction to Functional Analysis, John Wiley and sons, Inc., New York, 1958.

[11] Wang, D., Löwner-Heinz and Reid inequalities, Math. Japon, 49 (1999), 247-250. 